\title{
Comparison of Dissolution Profiles of Furosemide Tablets Available in the Argentinian Market
}

\author{
Yong K. Han, Laura D. Simionato, Romina G. Calvo, María B. Mattei and Adriana I. Segall \\ Cátedra de Calidad de Medicamentos, Facultad de Farmacia y Bioquímica. Universidad de Buenos Aires, \\ CONICET, Junín 956 (1113) CABA, Argentina
}

\begin{abstract}
In this work dissolution profiles of furosemide tablets of nine commercial products marketed in Argentine were evaluated. All brands fulfill the specifications of dissolution test of USP. Comparison of dissolution profiles were carried out by model-dependent and model independent approaches. Results obtained via model-dependent approach show a first order drug release mechanism especially for Brand I (reference) and Brand IV. Results obtained via modelindependent approach show that there was not significant difference in Dissolution efficiency between the reference product and Brands II, III and IV and in Mean dissolution time between the reference product and Brands II, III, IV and V. Using fit factors, only Brands I and III were similar.
\end{abstract}

Keywords: Furosemide, tablets, dissolution profiles, commercial products, model-dependent, model-independent.

\section{INTRODUCTION}

The rate and the extent of drug dissolution and its absorption depend on the characteristics of the active pharmaceutical ingredient (API) as well as the dosage form properties. Since an orally administered drug must be in solution in order to be absorbed in the gastro intestinal tract and to reach the systemic circulation, the dosage form plays an important role in condition the absorption rate [1].

Dissolution test are widely used in the pharmaceutical industry for developing new drug products, determining the long-term stability and shelf life of a dosage form and assessing the impact of postapproval changes in the manufacturing process. In the case of immediate-release solid dosage forms such as tablets, dissolution test are used to evaluate batches and can be used to evaluate new and existing formulations and possibly to assess the impact of certain changes in the formulation and manufacturing process [2]. Based on these consideration, dissolution test are largely used to assure the quality of the pharmaceutical product. Due to economic reasons, the use of generic medicines has been given much incentive by health authorities through the world. In our Country, there is a Disposition of our Health Authorities to evaluate bioavailability and bioequivalence of the market pharmaceutical products containing Furosemide [3]. Up to now, there have been no studies about bioavailability and bioequivalence of the marketed products.

*Address correspondence to this author at the Cátedra de Calidad de Medicamentos, Facultad de Farmacia y Bioquímica, Universidad de Buenos Aires, Junín 956 (1113) CABA, Argentina; Tel: 54-11-45083643;

E-mail: asegall@ffyb.uba.ar
Furosemide, 4-chloro-2-[(2-furanylmethyl)-amino]-5sulfamoylbenzoic acid, is a loop diuretic that is used orally in the treatment of edematous states associated with cardiac, renal, and hepatic failure and the treatment of hypertension. The usual dose is $40-120$ $\mathrm{mg} /$ day. Furosemide is a weak acid with an acidic $\mathrm{pKa}$ value of 3.8 (carboxylic acid). Seven polymorphic forms are known: four true polymorphs (I, II, III, and IV), two solvates (IV-DMS and V-dioxane) and one amorphous form but polymorph-dependent bioavailability has not been reported to date in the literature [4].

The available data on solubility, oral absorption, and permeability are sufficiently to classify furosemide into Class IV of the Biopharmaceutics Classification System (BCS4) [4]. A number of investigations have been done to improve furosemide solubility which can significantly increase its in vitro dissolution rate [5-16].

Although immediate release solid dosage forms are routinely subjected to test such as content uniformity, weight, hardness, friability and disintegration, the test that is most often associated with the assessment of in vivo performance is the dissolution test. Methods for comparing in vitro dissolution profiles can be classified into three main groups: ANOVA-based statistical methods, model-independent and model-dependent approaches. ANOVA-based methods can be classified as one way analysis of variance (ANOVA) and multivariate analysis of variance (MANOVA), which assess the difference between the means of two drug release data sets at a single point of dissolution and at multiple time points of dissolution, respectively [2]. Many studies have shown that ANOVA-based methods were overly discriminating and that it was difficult to distinguish between two dissolution curves [17-19]. 
All the model-dependent methods use some kind of curve fitting procedure. Different mathematical fit functions were investigated: the zero and first-order, Hixson-Crowell, Higuchi, quadratic, Weinbull, Gompertz and logistic [2, 17-19]. Model-dependent methods investigate the mathematical equations that describe the release profile in function of some parameters related to the pharmaceutical dosage forms so the quantitative interpretation of the values is easier. These methods seem to be useful in the formulationdevelopment stage.

The model-independent approaches produce a single value from a dissolution profile, providing direct comparisons of the dissolution data. Consequently the results do not depend on the selection of the specific parameter for fitting data but on the chosen sampling time. The model-independent methods include ratio test and the fit factors. Ratio test are performed as ratios of percent drug dissolved, area under the dissolution curve (AUC), and mean dissolution times (MDT) of the reference formulation with those of a test formulation at the same sampling time. Moore and Flanner [20] developed a simple model independent approach using fit factors. Fit factors include a difference factor $f 1$, and a similarity factor 12 . These fit factors compare the difference between the percent drug dissolved per unit time for a test and a reference formulation. Fit factors were adopted by FDA Center for Drug Evaluation and Research (CDER) [21] and the similarity factor was also adopted by the European Medicines Agency (EMA) Committee for Medicinal Products for Human Use (CHMP) [22] as an assessment criterion of similarity between two in vitro dissolution profiles. In Argentine, there is a Disposition to evaluate changes in the post marketing stage and to evaluate dissolution profiles using similarity factor $\mathfrak{2}$ [23].

In our country, Maggio et al. have studied eight lots of Furosemide tablets of three different brands. Brand $A$ was considered the innovator. The lots of Brands $B$ and $C$ complied the requirements for the evaluation of similarity and difference respect with Brand $A$, although there was difference between them [24]. Also, Ruiz et al, studied eleven Brands of our market. Compared with Brand A (reference product), there were three brands which differ in the similarity factor [25].

The aim of the present study was to evaluate and compare the dissolution profile of nine commercial products containing Furosemide $40 \mathrm{mg}$. marketed in Argentina, on the basis of their in vitro dissolution characteristics using USP, Test 1, Apparatus 2. Each formulation was compared with the reference using model-dependent methods: the zero and first-order and model-independent methods: fit factors, mean dissolution time (MDT) and dissolution efficiency \% (DE).

\section{MATERIALS AND METHODS}

\section{Reagents}

Analytical grade monobasic potassium phosphate (Anedra, Argentine) and sodium hydroxide (Mallinckrodt, USA) were used.

Furosemide was purchased in Saporiti (Argentine), $99.88 \%$, calculated with reference to the dried substance, origin India

\section{Materials}

In our study, nine commercial tablets containing Furosemide $40 \mathrm{mg}$ were purchased from pharmacies in Buenos Aires (Argentina). All tests were performed within products expiration dates.

\section{Apparatus and Procedure}

All dissolution studies were performed using USP37, Test 1 [26] Apparatus 2 in a Sotax AT7 (Sotax AG, Basel Switzerland), which is a manual-sampling dissolution bath. The furosemide tablets test was performed at $50 \pm 1 \mathrm{rpm}$. The dissolution medium was monobasic potassium phosphate $\mathrm{pH}$ : 5.8 at $37 \pm 0.5$ ${ }^{\circ} \mathrm{C}$. The acceptance criterion set was $\mathrm{Q}=80$ in $60 \mathrm{~min}$ (Test 1).

Dissolution media volume was $900 \mathrm{ml}$. In all experiments, $5 \mathrm{ml}$ sample aliquots were withdrawn at 5 , $15,30,45$ and $60 \mathrm{~min}$ using micropipettes. The withdrawn amounts were adjusted in the calculations. All samples were filtered through filter paper (Whatman $91 ; 10.0 \mu \mathrm{m})$. The filter paper used was properly validated using the standard solution and comparing with membrane filters. The amount dissolved was determined spectrophotometrically in a UV-VIS Spectrophotometer Cary 1E Varian (Victoria, Australia) at $274 \mathrm{~nm}$.

Twelve tablets or capsules of each preparation were studied to obtain statistically significant results.

\section{Comparative Dissolution}

\section{Model-Dependent Methods}

Mathematical models have been used extensively for the parametric representation of dissolution data. 
Furosemide release kinetics was analyzed by different mathematical models included zero order and first order, considering the amounts of drug release up to $60 \mathrm{~min}$.

Zero order: $Q_{t}=Q_{0}+K_{0} t$

First order: $\ln Q_{t}=\ln Q_{0}+K_{0} t$

\section{Model-Independent Methods}

\section{Fit Factors}

A mathematical comparison was performed by applying $f_{1}$ and $f_{2}$. These fit factors directly compare the difference between the percent drug dissolved per unit time for a test and a reference formulation.

$$
\begin{aligned}
& f 1=\left\{\frac{\left[\sum|R t-T t|\right]}{[\Sigma[R t]}\right\} \times 100, \text { where } t=1 \text { to } n \\
& f 2=50 \log \left\{\left[1+\frac{1}{n} \sum_{t=1}^{n}\left[(R t-T t)^{2}\right]\right]^{-0.5} \times 100\right\}
\end{aligned}
$$

(3) Difference factor

(4) Similarity factor

Where $n$ is the number of time points, $R_{t}$ is the dissolution value of the reference formulation at time $t$ and $T_{t}$ is the dissolution value of the test formulation at time $t$.

The similarity factor $\left(f_{2}\right.$.) is a logarithmic reciprocal square root transformation of the sum of squared error and is a measurement of the similarity in the percent (\%) dissolution between the curves. Values of $f_{1}$ between 0 and 15 and values of $f_{2}$ between 50 and 100 are used to define equivalence of two dissolution profiles, which means an average difference of no more than $10 \%$ at the sample time points.

\section{Dissolution Efficiency}

This concept was proposed by Khan and Rhodes in 1975 [27] and is defined as follows:

$$
D E \%=\frac{A U C_{0}^{T}}{Q_{100 . T}} .100
$$

Where $Q_{100}$ is the percentage of dissolved product, $D E$ is then the area under the dissolution curve between time points 0 and $T$ expressed as a percentage of the curve at maximum dissolution, $Q_{100}$, over the same time period.

\section{Mean Dissolution Time}

The mean dissolution time is calculated from the accumulative curves of dissolved product depending on the time [28].

$M D T=\frac{\sum[t i . \Delta Q i]}{Q_{\infty}}$

Where $t_{i}$ is intermediate time of the intervals of time sampled, $\Delta Q_{i}$ is the increase of the quantities of product dissolved in every interval of $t$ considered and $Q_{\infty}$ is the maximum of product dissolved.

The results of DE and MDT of the different Brands of furosemide tablets were compared with the reference using a two-variable $t$ test as follows:

$t=\frac{\left|\bar{X}_{R}-\bar{X}_{T}\right|}{S_{d}} \sqrt{\frac{1}{n_{R}}+\frac{1}{n_{T}}}$

where $\bar{X}_{R}$ and $\bar{X}_{T}$ are means of the model parameters of the reference and test products, respectively, $n_{R}$ and $n_{T}$ are the number of measurements for the mean $\bar{X}_{R}$ and $\bar{X}_{T}$, and $S_{d}$ is the weighted average standard deviation as shown below

$S_{d}=\sqrt{\frac{\left(n_{R}-1\right) S_{R+}^{2}\left(n_{T}-1\right) S_{T}^{2}}{n_{R}+n_{T}-2}}$

Where $S_{R}$ and $S_{T}$ are the standard deviation of the model parameters for the reference and test products. If the calculated $t$ values are lower than the critical value of $\mathrm{t}\left(1-\mathrm{\alpha} / 2, n_{R}+n_{T}-2\right)$, the two means $\bar{X}_{R}$ and $\bar{X}_{T}$ differ only randomly at risk level $\alpha$.

\section{RESULTS AND DISCUSSION}

Dissolution of drug from oral solid dosage forms is a necessary criterion for drug bioavailability (i.e. the drug must be solubilized in the aqueous environment of the gastrointestinal tract to be absorbed). For this reason, dissolution testing of solid oral drug products has emerged as one of the most important performance test for assuring product uniformity and batch to batch equivalence. Variations of the pharmacopeia limits indicate unacceptable products [21, 22].

Table 1 summarizes the characteristics of the nine products. The products were purchased from pharmacies in Buenos Aires (Argentina). All tests were performed within products expiration dates, which were similar among brands. In this study we defined Brand I as the reference product. 
Table 1: Formulation Compositions

\begin{tabular}{|c|c|c|}
\hline Brand & Other Ingredients & Appearance \\
\hline I & $\begin{array}{l}\text { Maize starch, pregelatinized starch, magnesium stearate, lactose, } \\
\text { colloidal silicon dioxide, talc. }\end{array}$ & White, circular, with indented line in center \\
\hline II & $\begin{array}{c}\text { Lactose, microcrystalline cellulose, povidone, sodium dioctyl } \\
\text { sulfosuccinate, sodium croscarmellose, sodium lauryl sulfate, talc, } \\
\text { aerosil, magnesium stearate. }\end{array}$ & White, circular, with indented line in center \\
\hline III & Lactose, maize starch polyvinylpyrrolidone. & White, circular, with indented line in center \\
\hline IV & $\begin{array}{l}\text { Lactose monohydrate, maize starch, povidone K-30, sodium } \\
\text { starch glycolate, magnesium stearate. }\end{array}$ & White, circular, with indented line in center \\
\hline V & $\begin{array}{l}\text { Maize starch, colloidal silicon dioxide, magnesium stearate, } \\
\text { povidone K30, sodium starch glycolate, talc, red dye ponceau 4R. }\end{array}$ & Pink, circular, with indented line in center \\
\hline VI & $\begin{array}{l}\text { Lactose, sodium starch glycolate, talc, magnesium stearate, } \\
\text { coprocessed lactose and microcrystalline cellulose. }\end{array}$ & White, circular \\
\hline VII & $\begin{array}{l}\text { Lactose, microcrystalline cellulose, sodium bicarbonate, sodium } \\
\text { starch glycolate, magnesium stearate. }\end{array}$ & White, circular, with indented line in center \\
\hline VIII & $\begin{array}{l}\text { Coprocessed lactose and microcrystalline cellulose } 80, \text { sodium } \\
\text { croscarmellose, colloidal silicon dioxide, magnesium stearate. }\end{array}$ & White, circular, with indented line in center \\
\hline IX & Ludipress, magnesium stearate. & White, circular, with indented line in center \\
\hline
\end{tabular}

Table 2: Dissolution Data and Descriptive Statistics of nine Brands of Furosemide Tablets

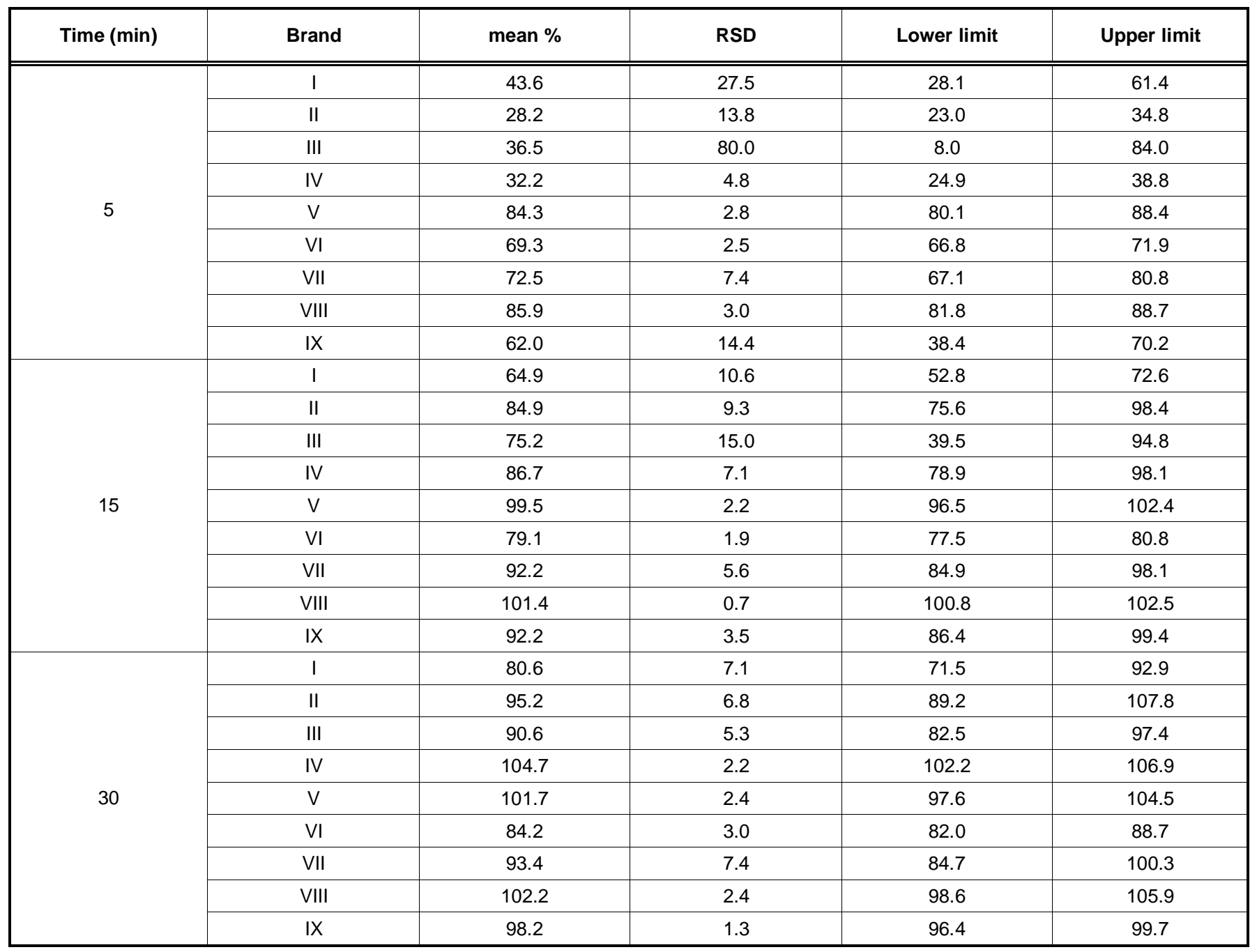


(Table 2). Continued.

\begin{tabular}{|c|c|c|c|c|c|}
\hline Time (min) & Brand & mean $\%$ & RSD & Lower limit & Upper limit \\
\hline \multirow{9}{*}{45} & I & 87.7 & 4.9 & 81.4 & 94.5 \\
\hline & II & 96.2 & 6.6 & 90.6 & 108.4 \\
\hline & III & 94.5 & 4.1 & 88.3 & 99.9 \\
\hline & IV & 107.1 & 1.5 & 104.4 & 109.0 \\
\hline & V & 104.1 & 1.1 & 103.0 & 106.0 \\
\hline & VI & 86.3 & 3.2 & 83.0 & 90.7 \\
\hline & VII & 93.8 & 7.8 & 85.4 & 102.2 \\
\hline & VIII & 103.3 & 1.6 & 102.2 & 106.1 \\
\hline & IX & 99.6 & 1.3 & 97.1 & 100.8 \\
\hline \multirow{9}{*}{60} & 1 & 92.4 & 3.2 & 89.0 & 99.2 \\
\hline & II & 96.6 & 5.1 & 92.4 & 105.3 \\
\hline & III & 95.1 & 3.6 & 90.4 & 99.4 \\
\hline & IV & 107.9 & 1.5 & 105.8 & 109.6 \\
\hline & V & 104.3 & 2.1 & 101.5 & 107.7 \\
\hline & VI & 87.7 & 3.0 & 84.2 & 90.2 \\
\hline & VII & 94.3 & 8.4 & 84.5 & 102.8 \\
\hline & VIII & 102.6 & 1.4 & 100.6 & 104.5 \\
\hline & IX & 99.6 & 1.8 & 97.7 & 102.6 \\
\hline
\end{tabular}

In the dissolution test for furosemide tablets described in the American Pharmacopeia (United States Pharmacopeia 37) no less than $85 \%(Q+5 \%)$ should be dissolved in 60 minutes. Table 2 summarizes the mean percent dissolved at each time point, the relative standard deviation (RSD), and the upper and lower limits.
Evaluating the dissolved percentage curves vs. time (Figure 1), it could be observed that the analyzed products presented very distinct dissolution profiles. As furosemide is a BCS IV drug, the dissolution test may be formulation dependent, and the decision related to generics must be made based on the in vivo bioequivalence results.

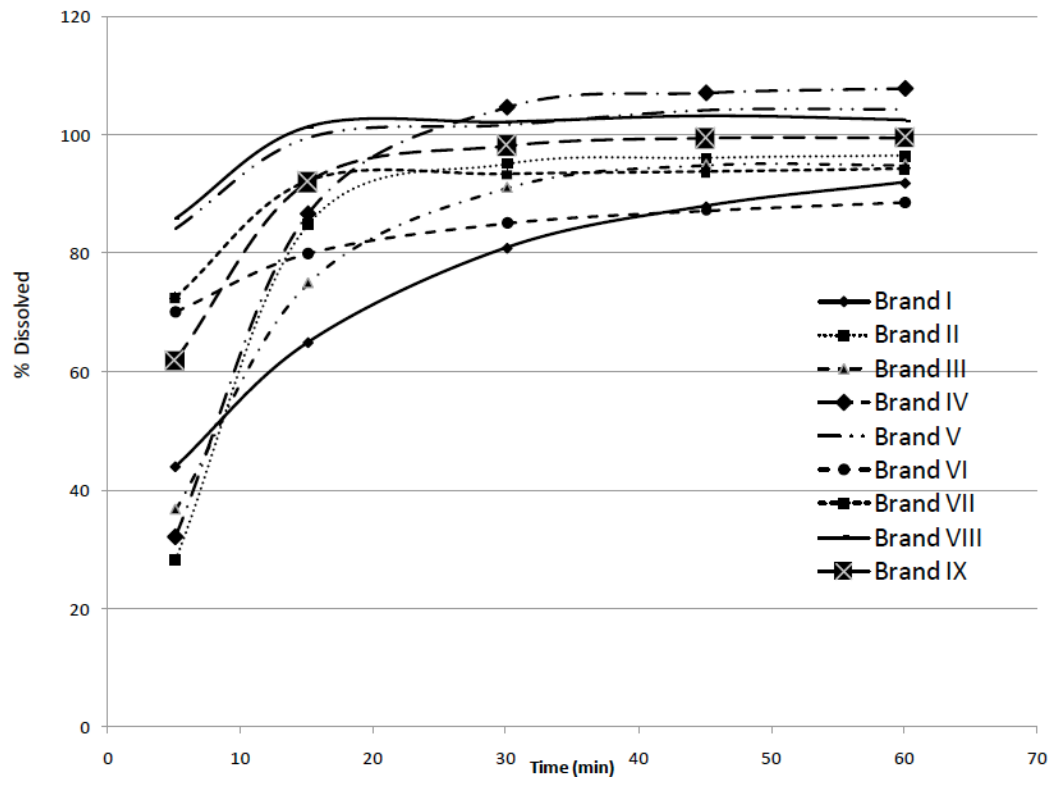

Figure 1: Dissolution profiles. 
Table 3: Linearization of the Furosemide Release Profiles (Q Expressed in $\mathrm{mg}$ )

\begin{tabular}{|c|c|c|c|c|}
\hline \multirow{2}{*}{ Brand } & \multicolumn{2}{|c|}{ Zero order } & \multicolumn{2}{c|}{ First order } \\
\cline { 2 - 5 } & $\mathbf{K}(\mathbf{m g} / \mathbf{m i n})$ & $\mathbf{R}^{2}$ & $\left.\mathbf{R}^{2} \mathbf{m i n}^{-1}\right)$ & $\mathbf{0 . 9 9 3 3}$ \\
\hline \hline I & 0.3361 & 0.8824 & -0.0360 & 0.7930 \\
\hline II & 0.3983 & 0.5617 & -0.0520 & 0.9008 \\
\hline III & 0.3728 & 0.6990 & -0.0465 & 0.9965 \\
\hline IV & 0.4669 & 0.6455 & -0.1157 & 0.0360 \\
\hline V & 0.1210 & 0.6504 & -0.0107 & 0.9022 \\
\hline VI & 0.1220 & 0.8199 & -0.0158 & 0.5971 \\
\hline VII & 0.1204 & 0.5069 & -0.0227 & 0.6165 \\
\hline VIII & 0.0939 & 0.4958 & -0.0565 & 0.9197 \\
\hline IX & 0.2281 & 0.5838 & -0.0841 & \\
\hline
\end{tabular}

Dissolution profiles of the nine products were evaluated by fitting experimental data to zero and first kinetics order. Table 3 lists zero and first order dissolution constants $(\mathrm{K})$ and determination constant $\left(r^{2}\right)$ values, bold print indicating the best fits. For the nine products the curve of better adjustment was the first order. Brands 1 and IV have the best fitting, with the maximum determination coefficient. The results of the first order model for the nine brands indicate different drug release mechanism for the furosemide products.

The DE and the MDT values are a useful way to reduce each curve to a single number, which may be related to the dissolution rate constant. The average, the standard deviation (SD), the variation coefficient (CV) and tex of the DE data are presented in Table 4. Test " $\mathrm{f}$ " with $95 \%$ confidence for 22 degrees of freedom was $\left(t_{n-2}, \alpha: 0.05\right)=2.0739$. There was no significant difference between the reference product and Brands II, III and IV.The average, SD, CV and $t_{\text {ex }}$ of the MDT data are presented in Table 5. Test " $t$ " with 95\% confidence for 22 degrees of freedom was $\left(t_{n-2, \alpha: 0.05}\right)=$ 2.0739. There was no significant difference between the reference product and Brands II, III, IV and V.

Fit factors are important quantitative methods that have been recommended by FDA guidelines for industry for comparison of dissolution profiles [21]. Results obtained from the test using Brand $I$ as the reference are shown in Table 6 . The similarity factor $f_{2}$ is more sensitive in finding dissimilarity between dissolution curves than the difference factor $f_{1}$, and the values of fit factors are dependent on the number of sampling time point chosen. According to FDA $f_{1}$ values up to 15 and $f_{2}$ values greater than 50 should ensure equivalence of the dissolution curves, indicating an average difference of no more than $10 \%$ at the sample time points. Based on this guideline, only Brand III seems to show a dissolution curve similar with the reference.

Table 4: Average (M), Standard Deviation (SD), Variation Coefficient (CV) and $t$ Experimental ( $t_{\mathrm{ex}}$ ) of Dissolution Efficiency \% (ED)

\begin{tabular}{|c|c|c|c|c|}
\hline Brand & M & SD & CV & $\boldsymbol{t}_{\text {ex }}$ \\
\hline \hline I & 74.4 & 2.9 & 3.8 & 1.6293 \\
\hline II & 83.9 & 1.8 & 10.3 & 0.3851 \\
\hline III & 80.2 & 8.2 & 2.8 & 1.2116 \\
\hline IV & 82.1 & 2.3 & 1.8 & 3.0707 \\
\hline V & 91.8 & 1.6 & 1.6 & 2.7727 \\
\hline VI & 89.8 & 1.4 & 1.7 & 3.2399 \\
\hline VII & 92.7 & 1.6 & 0.7 & 3.8096 \\
\hline VIII & 93.7 & 0.6 & 3.2 & 2.1882 \\
\hline IX & 89.8 & 2.9 & & \\
\hline
\end{tabular}


Table 5: Average (M), Standard Deviation (SD), Variation Coefficient (CV) and $t$ Experimental ( $\left.t_{\mathrm{ex}}\right)$ of Mean Dissolution Time (MDT)

\begin{tabular}{|c|c|c|c|c|}
\hline Brand & M & SD & CV & ex \\
\hline \hline I & 20.4 & 2.0 & 9.6 & 1.5382 \\
\hline II & 14.2 & 1.2 & 36.3 & 0.3244 \\
\hline III & 16.8 & 6.1 & 8.7 & 1.5382 \\
\hline IV & 14.2 & 1.2 & 10.4 & 1.1124 \\
\hline V & 15.5 & 1.6 & 10.1 & 2.9185 \\
\hline VI & 9.4 & 1.0 & 14.0 & 3.3838 \\
\hline VII & 7.4 & 1.0 & 6.6 & 3.9479 \\
\hline VIII & 6.7 & 0.4 & 1.6 & 2.3430 \\
\hline
\end{tabular}

Table 6: Fit Factors for the Nine Brands of Furosemide Tablets Based on The Average of Twelve Tablets

\begin{tabular}{|c|c|c|}
\hline \multirow{2}{*}{ Brand } & \multicolumn{2}{|c|}{ Fit Factor } \\
\cline { 2 - 3 } & $\mathbf{f}_{\mathbf{1}}$ & $\mathbf{f}_{\mathbf{2}}$ \\
\hline \hline $\mathrm{I} / \mathrm{II}$ & 17.0 & 43.1 \\
\hline $\mathrm{I} / \mathrm{III}$ & $\mathbf{1 0 . 0}$ & $\mathbf{5 4 . 3}$ \\
\hline $\mathrm{I} / \mathrm{IV}$ & 25.3 & 36.1 \\
\hline $\mathrm{I} / \mathrm{V}$ & 33.8 & 28.4 \\
\hline $\mathrm{I} / \mathrm{VI}$ & 10.2 & 43.9 \\
\hline $\mathrm{I} / \mathrm{VII}$ & 20.9 & 36.4 \\
\hline $\mathrm{I} / \mathrm{VIII}$ & 34.2 & 27.7 \\
\hline $\mathrm{I} / \mathrm{IX}$ & 22.3 & 37.7 \\
\hline
\end{tabular}

\section{CONCLUSION}

This study found variations in the dissolution profiles of furosemide tablets commonly available in Argentina. The analyzed products presented very distinct dissolution profiles, showing that the dissolution test may be formulation dependent. All Brands fulfill the specifications of dissolution test of USP 37.

The kinetic curve of better adjustment was the first order. Brands I and IV have the best fitting, with the maximum determination coefficient. There is no significant difference in DE among the reference product and Brands II, III and IV. There is not significative difference in MDT between the reference product and Brands II, III, IV and V.

Using fit factors, only Brands I and III were similar.

In conclusion, significant differences were seen between the in vitro dissolution profiles of furosemide tablets from various commercial preparations. Unless, it has been demonstrated in vivo bioequivalence of furosemide market products interchangeability with generics should be avoided.

\section{ACKNOWLEDGEMENT}

This work was supported by grant 20020100100816 from UBA and PIP №: 11420110100380 from CONICET both to A. I. Segall.

\section{REFERENCES}

[1] Perioli L, D'Alba G, Pagano C. New oral solid dosage form for furosemide oral administration. Eur $\mathrm{J}$ Pharm Biopharm 2012; 80: 621-9.

http://dx.doi.org/10.1016/j.ejpb.2011.12.011

[2] Khan F, Li M, Schlindwein W. Comparison of in vitro dissolution tests for commercially available aspirin tablets. Diss Technol 2013; 20: 48-58.

[3] Disposición № 4788 (ANMAT), Buenos Aires, Argentina, 15 de Agosto de 2012.

[4] Granero GE, Longhi MR, Mora MJ, et al. Biowaiver monographs for immediate release solid oral dosage forms: Furosemide. J Pharm Sci 2010; 99: 2544-56. http://dx.doi.org/10.1002/jps.22030

[5] Doherty C, York P. Evidence for solid- and liquid-state interactions in a furosemide-polyvinylpyrrolidone solid dispersion. J Pharm Sci 1987; 76: 731-7. http://dx.doi.org/10.1002/jps.2600760912

[6] Akbuga J, Gürsoy A, Yetimoglu F. Preparation and properties of tablets prepared from furosemide-PVP solid dispersion systems. Drug Dev Ind Pharm 1988; 14: 2091108.

http://dx.doi.org/10.3109/03639048809152004

[7] Ozdemir N, Ordu S. Improvement of dissolution properties of furosemide by complexation with beta-cyclodextrin. Drug Dev Ind Pharm 1998; 24: 19-25. http://dx.doi.org/10.3109/03639049809082348

[8] Shin SC, Oh IJ, Lee YB, Choi HK, Choi JS. Enhanced dissolution of furosemide by coprecipitating or cogrinding with crospovidone. Int J Pharm 1998; 175: 17-24. http://dx.doi.org/10.1016/S0378-5173(98)00260-9 
[9] Shin SC, Kim J. Physicochemical characterization of solid dispersion of furosemide with TPGS. Int J Pharm 2003; 251: 79-84. http://dx.doi.org/10.1016/S0378-5173(02)00586-0

[10] Farcas A, Jarroux N, Farcas AM, Harabagiu V, Guegan P. Synthesis and characterization of furosemid complex in bcyclodextrin. Dig J Nanomater Bios 2006; 1: 55-60.

[11] Kaukonen AM, Laitinen L, Salonen J, et al. Enhanced in vitro permeation of furosemide loaded into thermally carbonized mesoporous silicon (TCPSi) microparticles. Eur J Pharm Biopharm 2007; 66: 348-56. http://dx.doi.org/10.1016/j.ejpb.2006.11.021

[12] Patel RP, Patel DJ, Bhimani DP, Patel JK. Physicochemical characterization and dissolution study of solid dispersions of furosemide with polyethylene glycol 6000 and polyvinyl pyrrolidone K30. Diss Technol 2008; 15: 17-25.

[13] Chaulang G, Patel P, Hardikar S, Kelkar M, Bhosale A, Bhise $S$. Formulation and evaluation of solid dispersions of furosemide in sodium starch glycolate. Trop J Pharm Res 2009; 8: 43-51. http://dx.doi.org/10.4314/tipr.v8i1.14711

[14] Zvonar A, Berginc K, Kristl A, Gašperlin M. Microencapsulation of self-microemulsifying system: improving solubility and permeability of furosemide. Int $\mathrm{J}$ Pharm 2010; 388: 151-8. http://dx.doi.org/10.1016/j.ijpharm.2009.12.055

[15] Perioli L, Ambrogi V, Nocchetti M, Sisani M, Pagano C. Preformulation studies on host-guest composites for oral administration of BCS class IV drugs: HTlc and furosemide. Appl Clay Sci 2011; 53: 696-703. http://dx.doi.org/10.1016/j.clay.2011.06.006

[16] De Zordi N, Monaghan M, Kikic I, et al. Applications of supercritical fluids to enhance the dissolution behaviors of Furosemide by generation of microparticles and solid dispersions. Eur J Pharm Biopharm 2012; 81: 131-41. http://dx.doi.org/10.1016/j.ejpb.2012.01.002

[17] Yuksel N, Kanik AE, Baykara T. Comparison of in vitro dissolution profiles by ANOVA-based, model-dependent and -independent methods. Int J Pharm 2000; 209: 57-67. http://dx.doi.org/10.1016/S0378-5173(00)00554-8
[18] Polli JE, Rekhi GS, Ausburger LL, Shah VP. Methods to compare dissolution profiles and a rationale for wide dissolution specifications for metoprolol tartrate tablets. J Pharm Sci 1997; 86: 690-700.

http://dx.doi.org/10.1021/js960473x

[19] Costa FO, Sousa JJS, Pais AACC, Formosinho SJ. Comparison of dissolution profiles of lbuprofen pellets. $J$ Control Release 2003; 89: 199-212. http://dx.doi.org/10.1016/S0168-3659(03)00033-6

[20] Moore JW, Flanner HH. Mathematical comparison of dissolution profiles. Pharm Technol 1996; 20: 64-74.

[21] Guidance for the industry: dissolution testing of immediate release solid oral dosage forms, FDA, Center for Drug Evaluation and Research, Rockville, MD, 1997.

[22] Guideline on the investigation of bioequivalence: EMA Committee for Medicinal Products for Human Use (CHMP), London, UK, 2010.

[23] Disposición No 5568 (ANMAT), Buenos Aires, Argentina, 5 de Febrero de 2009.

[24] Maggio RM, Castellano PM, Kaufman TS. A new principal component analysis-based approach for testing "similarity" of drug dissolution profiles. Eur J Pharm Sci 2008; 34: 66-77. http://dx.doi.org/10.1016/j.ejps.2008.02.009

[25] Ruiz ME, Gregorini A, Talevi A, Volonté MG. Dissolution studies of generic medications: New evidence of deviations from the Transitivity principle. Diss Technol 2012; 19: 13-24.

[26] The United States Pharmacopeia $37^{\text {th }}$ Ed.) U.S. Pharmacopeial Convention, Rockville, MD; 2014, 3104-3105.

[27] Khan KA, Rhodes CT. Effect of compaction pressure on the dissolution efficiency of direct compression systems. Pharm Acta Helv 1972; 47: 594-607.

[28] Aguilar Ros A, Caamaño Somoza M, Martín Martín FR, Montejo Rubio MC. Parámetros amodelísticos: comparación de perfiles. In Biofarmacia y Farmacocinética, ejercicios y problemas resueltos, $1^{\text {st }}$ ed. Elsevier España S. L., Barcelona, España, 2008; pp. 3-4 